\title{
SELF-UNIVERSAL CRUMPLED CUBES AND A DOGBONE SPACE
}

\author{
E. H. ANDERSON ${ }^{1}$
}

\begin{abstract}
The question of whether each self-universal crumpled cube is universal is answered negatively by presenting an example of a dogbone space which is not topologically $E^{3}$ but which can be expressed as a sewing of two crumpled cubes, one of which is selfuniversal.
\end{abstract}

C. D. Bass and R. J. Daverman [2] presented a brief paper indicating that the solid Alexander horned sphere is an example of a crumpled cube which is self-universal but not universal, thus answering negatively the question asked by C. E. Burgess and J. W. Cannon in [4] of whether each self-universal crumpled cube is universal. The validity of the example presented by Bass and Daverman depends on a claim that a certain upper semicontinuous decomposition of $S^{3}$ into points and tame arcs, described in [2], is not topologically $S^{3}$, which in turn depends on the validity of four lemmas which are stated in $[2, \S 2]$. The proofs of these four lemmas appear to entail nontrivial arguments which are not included in [2].

In this note, we present an example of a dogbone space, an upper semicontinuous decomposition of $S^{3}$ into points and tame arcs whose nondegenerate elements can be expressed as the intersection of a tower of solid double tori, which is not topologically $S^{3}$. The dogbone space can be described as the result of a sewing of two crumpled cubes, one of which is self-universal. Thus, the question asked by Burgess and Cannon in [4] is answered negatively. The argument will be based essentially upon work by Casler [5] and the author [1].

Some recent work by Eaton [6] includes a different proof that the solid Alexander horned sphere $H$, used in the example presented by Bass and Daverman, is not universal. This was done by sewing $H$ to the crumpled cube $T$ described by Stallings [7] so that the wild points of Bd $H$ are sewn to the Cantor set of nonpiercing points of $T$. Other methods developed by Eaton, in papers cited in [6], should offer alternative ways

Presented to the Society, October 30, 1971; received by the editors August 18, 1971. AMS 1970 subject classifications. Primary 57A10.

$K e y$ words and phrases. Crumpled cube, upper semicontinuous decomposition, $E^{3}$.

${ }^{1}$ Supported by Mississippi State University Biological and Physical Sciences Research Institute.

c) American Mathematical Society 1972 
to show that neither the decomposition space we describe here nor the one described by Bass and Daverman [2] is topologically $S^{3}$.

Definitions relating to crumpled cubes will be those of [2].

1. The example. The description of the dogbone space of this paper is modeled after that by Bing in [3]. As in Figure 1, let $H_{1}$ and $H_{2}$ denote

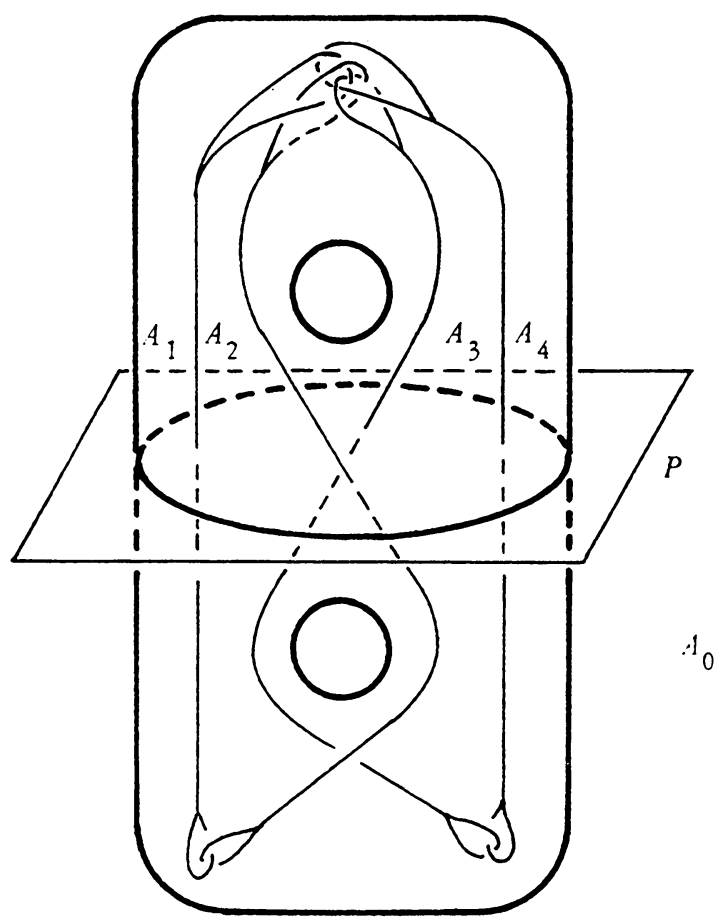

Figure 1

the upper and lower half-spaces of $S^{3}$, and $P$ the $x y$-plane. Let $A_{0}$ denote a solid double torus which intersects $P$ in a disk. Then, embed solid double tori $A_{1}, \cdots, A_{4}$, linked as indicated, in $A_{0}$; although each of $A_{1}, \cdots, A_{4}$ is shown as a finite graph, it is topologically equivalent to $A_{0}$. Then, for each $i=1, \cdots, 4$, solid double tori $A_{i, 1}, \cdots, A_{i .4}$ are embedded in $A_{1}$ such that there is a homeomorphism of $S^{3}$ onto itself which is the identity on the complement of some open set containing $A_{0}$ and takes $A_{0}$ onto $A_{i}$. Succeeding steps of the construction are to be described inductively.

Let $M=A_{0} \cap \sum A_{i} \cap \sum A_{i, j} \cap \sum A_{i, j, k} \cdots$. Let $G$ be the set whose elements are components of $M$ and one-point subsets of $S^{3}-M$. Then, $G$ is an upper semicontinuous decomposition of $S^{3}$ into points and tame 
arcs. Let $S^{3} / G$ denote the associated decomposition space, the dogbone space of this note.

From [1], we have that $S^{3} / G$ is not topologically $S^{3}$.

The methods of Casler [5] can be easily modified to show that $H_{2}^{*}$, the natural projection of $H_{2}$ in $S^{3} / G$, is a self-universal crumpled cube.

Since $H_{1}^{*}$, the natural projection of $H_{1}$ in $S^{3} / G$, is a crumpled cube and $S^{3} / G$ is the result of a sewing of $H_{1}^{*}$ and $H_{2}^{*}$, we have that $H_{2}^{*}$ is not a universal crumpled cube.

\section{BIBLIOGRAPHY}

1. E. H. Anderson, Chains of simple closed curves and a dogbone space, Fund. Math. 72 (1971), 131-144.

2. C. D. Bass and R. J. Daverman, A self-universal crumpled cube which is not universal, Bull. Amer. Math. Soc. 76 (1970), 740-742. MR 41 \#7655.

3. R. H. Bing, $A$ decomposition of $E^{3}$ into points and tame arcs such that the decomposition space is topologically different from $E^{3}$, Ann. of Math. (2) 65 (1957), 484-500. MR 19, 1187.

4. C. E. Burgess and J. W. Cannon, Embeddings of surfaces in $E^{3}$, Rocky Mt. J. Math. 1 (1971), 259-344. MR 43 \#4008.

5. B. G. Casler, On the sum of two solid Alexander horned spheres, Trans. Amer. Math. Soc. 116 (1965), 135-150. MR 32 \#3049.

6. W. T. Eaton, The sum of solid spheres, Michigan Math. J. (to appear).

7. J. R. Stallings, Uncountably many wild disks, Ann. of Math. (2) 71 (1960), 185-186. MR 22 \#1871.

Department of Mathematics, Mississippi State University, State College, MisSISSIPPI 39762 